\title{
THE TRANSITION FROM THE IONOSPHERE TO INTERPLANETARY SPACE
}

\begin{abstract}
$\mathrm{D}$ URING the past decade there has been a revival of interest in the problems of the outer corona (commonly called the zodiacal light region) and in the study of the outer ionosphere. For the ionosphere this interest has been increased by three factors. The original work of L. R. O. Storey on whistling atmospherics has been developed to give reasonably reliable values of electron density at distances of up to four Earth radii from the Earth. It is now possible to obtain strong radio reflexions from the Moon, giving information about the electron content of the upper ionosphere. Lastly, observation of the radio emission from artificial Earth satellites is likely to provide data concerning electron density and irregular ties above the $F 2$ peak of the ionosphere.
\end{abstract}

These matters formed the subject of a Geophysical Discussion held in the rooms of the Royal Astronomical Society at Burlington House on February 21 under the chairmanship of Mr. J. A. Rateliffe (Cambridge). The discussion was initiated by five short talks by astrophysicists and ionospheric physicists.

Prof. Sydney Chapman began by describing his model of the complete solar corona, extending from 0.06 solar radii above the solar photosphere to the boundaries of the known solar system at 40 A.U. (astron. units) or $8.6 \times 10^{3}$ solar radii. This is a static model consisting solely of ionized hydrogen in which there is a constant outward flow of heat by thermal conduction. The thermal conductivity of this gas can be calculated with reasonable accuracy from kinetic theory considerations, assuming no appreciable magnetic field. Taking the value of the electron density in the corona at 1.06 solar radii from eclipse measurements, and assuming a temperature in this region of about $10^{\prime}$ deg. K., both the temperature and the electron density can be calculated as a function of distance from the Sun. According to this theory the electron density at large distances is remarkably sensitive to the assumed temperature at the base of the corona. At 1 A.U. it changes from $3 \times 10^{2} \mathrm{~cm}^{-8}$ to $4 \times$ $10^{3} \mathrm{~cm}^{-3}$ for an increase in the assumed temperature at 1.06 solar radii from $1.0 \times 10^{6} \mathrm{deg}$. K. to $1.2 \times$ $10^{6} \mathrm{deg}$. $\mathrm{K}$. The calculated temperature is $2 \cdot 2 \times 10^{5}$ deg. at 1 A.U. and $7.7 \times 10^{4}$ deg. at 40 A.U. The calculated electron density values are in good agree. ment with the current eclipse values (which extend to 20 solar radii) and with the generally assumed values in the zodiacal light region. As the thermal conductivity of an ionized gas depends upon the magnetic field in the gas, these deduced values of electron density and temperature may be seriously affected by interplanetary fields of solar origin, although the good agreement with eclipse models to 20 solar radii shows that such fields may be unim. portant for the model. However, Prof. Chapman emphasized that his model is no more than a preliminary idealized static model, and in a real corona there are undoubtedly other mechanisms operating, particularly corpuscular emission from the Sun. An important feature of this theory is that as the Earth may be imagined as a cool body passing through a hot gas, there is a ready explanetion for the supposed downward flux of heat through the F2-layer.

The problem of the determination of electron density in interplanetary space by observation of the zodiacal light was discussed by Dr. D. E. Blackwell (Cambridge). The zodiacal light is seen in tropical regions as a cone of light standing above the western horizon after sunset, or the eastern horizon before sunrise. Its observation is peculiarly difficult, and the interpretation of the data is uncertain. The phenomenon is caused by the scattering of sunlight by interplanetary material, and its brightness at each place in the sky is the sum of contributions from scattering elements along the line of sight through the solar system. The active scattering material is now supposed to be partly dust and partly free electrons, and the first big problem is to decide the relative contributions of dust and electrons, and to determine electron densities. The only method of separation that has been tried so far has used observations of polarization. The polarization of the electron component can be calculated and that of the dust is assumed to be zero. Knowing the total polarization, the relative proportions of dust and electron components can be calculated. This method gives for the electron density at about 1 A.U. from the Earth a value of about $600 \mathrm{~cm} .^{-8}$, which is in apparent agreement with values obtained from whistling atmospherics by Storey and others. How. ever, acceptance of this value leads to difficulties. One is that the plane of symmetry of the electron component is very close to the ecliptic, which has gravitational significance only. Another is that it leads to two subsidiary conditions, one of which must be accepted. Either there is a sharp cut-off of electron density near 1 A.U. (corresponding to a sharp diminution of ionization) or else the polarization of the dust component is negative. Neither condition is welcome, and the simplest way out of the difficulty is to suppose that the electron component does not exist.

The information obtainable from the study of radio echoes from the Moon was discussed by Dr. J. V. Evans. Considering the propagation of a plane polarized wave through the ionosphere, the plane of polarization of the received wave is rotated by an amount proportional to

$$
\frac{1}{f^{2}} \int_{0}^{R} N_{e} \cdot B \cos \theta \sec \gamma . \mathrm{d} h
$$

where $N_{e}$ is the electron density, $B \cos \theta$ the component of the Earth's magnetic field along the ray path, $\chi$ the zenith angle, and $f$ the frequency of the wave. To obtain the number of complete revolutions of the plane of polarization it is necessary to observe simultaneously at two frequencies, those used by Evans and his collaborators being $119.28 \mathrm{Mc} / \mathrm{s}$. and $120.72 \mathrm{Mc} / \mathrm{s}$. Measurements of the total rotation can be used to check an ionospheric model. In such a model, most of the rotation occurs below a height 
of $600 \mathrm{~km}$. because the function $B \cos \theta \sec \chi$ decreases rapidly beyond this height. If an average value for $B \cos \theta$ sec $\chi$ is used up to a height of $600 \mathrm{~km}$., the observed rotation gives the total electron content of the ionosphere. Results obtained over a period of a year suggest that the number of electrons above the F2-region, at a height of about $350 \mathrm{~km}$., is about three times as great as the number below. The rotation produced by the ionosphere is variable and it would be very difficult to measure the small residual rotation produced by the electrons between the Moon and the ionosphere. It is suggested that a comparison of the total electron content deduced from Moon-echo work with that deduced from observation of an artificial Earth satellite might give this density.

Dr. K. Weekes (Cambridge) discussed the deductions about the outer ionosphere that may be made from observations of artificial Earth satellites. One clear application is to the study of irregularities in the ionosphere. A study of the fluctuations of the signal from the first Russian satellite $\left(1957 \alpha_{1}\right)$ showed that the bottom of the irregular zone is at a height of $280 \mathrm{~km}$. More precise knowledge can be expected to follow from three kinds of measurement. These are, the Doppler effect, the Faraday effect or the rotation of the plane of polarization, and the angle of arrival of the radio signal. The first effect depends upon the refractive index of the medium, but is independent of the Earth's magnetic field. The second effect depends upon the magnetic field. The Doppler effect and the angle of arrival are closely related and give the same information unless very related ande measures are available, when it should be possible to deduce the electron density at the satellite. Such a deduction requires a measurement of the Doppler shift to $0.5 \mathrm{c} . / \mathrm{s}$. and the angle of arrival to $1 \mathrm{~min}$. of arc ; observations of Satellite $1957 \alpha_{1}$ were too inaccurate to permit such deductions. The Faraday effect can be separated from effects of rotation of the setellite by observing nearly simulrotation of the satellite by observing nearly simul effect should be independent of frequency, whereas the Faraday effect varies inversely as the square of the frequency. It is usually very difficult to separate the frequency. It is of scintillation, but data from the few good records available are consistent with the known ionosphere and show that the electron density falls off rather slowly above the F2-layer.

Some recent American work on audio-frequency atmospherics, particularly that of Helliwell at Stanford and Gallet at the U.S. Bureau of Standards, was summarized by Mr. J. A. Ratcliffe. These atmospheries were first thoroughly investigated by Storey at Cambridge, who showed that each burst is propagated along a line of force of the Earth's magnetic field, the musical note resulting from dispersion in the ionosphere. This theory requires the electron density at a distance of 3 Farth radii to be about $300 \mathrm{~cm}^{-8}$. Since Storey's pioneering work, the time variation of frequency of the received atmospheric has been studied towards higher frequencies, and it has been found possible to deduce from the shape of the curve the magnetic field at the maximum height of the line of force. As may be expected, this maximum height depends upon the geomagnetic latitude of the source of the atmospherics, the grestest height being reached for those lines of force beginning and ending in high latitudes. This maximum height is about 4 Earth radii. How. ever, more recent work has shown that some whistlers are multiple, which suggests that the Earth's magnetic field is not the sole guiding factor. It is possible that the multiple effect is due to a streaky ionosphere, the waves being to some extent guided by filamentary structures which are roughly aligned along the lines of force.

There is also a second type of naturally occurring audio-frequency atmospheric which is characterized by $a$ different variation of frequency with time, showing a minimum frequency. It has been suggested that this type of atmospheric is excited in the ionosphere by corpuscular radiation from the Sun. For this mechanism to operate, the corpuscular velocity at the Earth must be of the order of $10^{4} \mathrm{~km}$. sec.-1, which means that the particles are probably accelerated near the Earth.

At this stage the chairman invited discussion of these topics. Dr. A. Hewish described the Cambridge work on the occultation of radio sources by the solar corona. Scattering of the incident radio wave by irregularities in the corona can be observed to a distance of 20 solar radii. Such irregularities are difficult to explain unless the general magnetic field of the Sun extends to distances at least as great as this, and consequently the thermal conductivity may be appreciably smaller than that assumed by Prof. Chapman. An elementary consideration of rotational stability suggests that such a field could not extend to distances greater than about $\mathbf{4 0}$ solar radii if it is of a dipole character.

Dr. R. d'E. Atkinson (Royal Observatory) thought that a corona of the Chapman type would considerably damp the orbital motion of the Earth, but Prof. Chapman again emphasized that his is a very idealized model and undoubtedly the real corona is far more complex.

Prof. C. W. Allen (University College, London) suggested that the solar magnetic field a few radii from the Sun is directed towards the Earth. If this is correct, the Chapman theory is not substantially changed by the inclusion of an interplanetary mag. netic field. Prof. V. C. A. Ferraro agreed with Prof. Allen on this point.

Mr. Ratcliffe stressed the apparent disegreement between the optical results, which suggest a very small electron density, and the whistler results, which suggest a density between 600 and $1,000 \mathrm{~cm}^{-\mathrm{s}}$. He asked if the Chapman theory predicts an annual variation in the ionosphere due to the variation of the distance of the Sun.

In reply, Dr. Blackwell said that the apparent disagreement between the radio and optical results may only be due to an unexpectedly great extension of the Earth's atmosphere. Recent Russian observations of the enhancement of the 5577 OI atmospheric emission line in the zodiacal light support this theory.

Prof. Chapman could not say definitely whether or not the changing distance of the Sun would have an effect on the ionosphere. He thought that acceptance of his theory does not depend upon the zodiacal light measures of electron density.

Prof. Allen thought there was little essential difference between the static corona of Chapman and dynamic theories. Dr. R. A. Lyttleton (Cambridge) considered that the sudden disruption of comet tails indicated the presence of interplanetary gas.

This discussion has demonstrated that important results may be expected to follow from a combination of astronomical and ionospheric techniques.

D. E. BLACKWELL 